\title{
REVERSED PRESERVATION PROPERTIES FOR SERIES AND PARALLEL SYSTEMS
}

\author{
FÉLIX BELZUNCE, ${ }^{* * *}$ Universidad de Murcia \\ HELENA MARTÍNEZ-PUERTAS, ${ }^{* * *}$ Universidad de Almería \\ JOSÉ M. RUIZ, ${ }^{* * * * *}$ Universidad de Murcia
}

\begin{abstract}
Recently Li and Yam (2005) studied which ageing properties for series and parallel systems are inherited for the components. In this paper we provide new results for the increasing convex and concave orders, the increasing mean residual life (IMRL), decreasing failure rate (DFR), the new worse than used in expectation (NWUE), the increasing failure rate in average (IFRA), the decreasing failure rate in average (DFRA), and the new better than used in the convex order (NBUC) ageing classes.
\end{abstract}

Keywords: Increasing convex order; increasing concave order; IMRL; DFR; NWUE; IFRA; DFRA; NBUC; parallel and series system

2000 Mathematics Subject Classification: Primary 60E15

Secondary $60 \mathrm{~K} 10$

\section{Introduction}

One of the main problems in reliability theory is the study of ageing properties of a coherent system from ageing properties of the components. For example, Esary et al. (1970) and Ross (1972) proved that for a coherent system with independent components, if the components are new better than used (NBU) or IFRA then the system is NBU or IFRA, respectively. For some particular coherent systems, which include series and parallel systems, Esary and Proschan (1963) proved a similar result for the increasing failure rate property, and Franco et al. (2003) proved some results for the increasing likelihood ratio (ILR) and the decreasing likelihood ratio (DLR) properties. Some extensions of these results can be found in Belzunce et al. (2002). However, it is not so easy to provide similar preservation results for some other ageing classes and some papers have been devoted to the study of particular coherent systems such as parallel and series systems. For example, Abouammoh and El-Neweihi (1986) proved that the decreasing mean residual life (DMRL) and the new better than used in expectation (NBUE) ageing classes are preserved under the formation of parallel systems with independent and identically distributed (i.i.d.) random variables, and Hendi et al. (1993) proved a similar result for the NBUC ageing property. Li et al. (2000) and Pellerey and Petakos (2002) extended this result for the case of parallel systems with independent and not necessarily identically distributed components. Some other results for

\footnotetext{
Received 23 May 2007; revision received 12 September 2007.

* Postal address: Departamento Estadística e Investigación Operativa, Universidad de Murcia, Campus de Espinardo, 30100 Espinardo de Murcia, Spain.

** Email address: belzunce@um.es

*** Postal address: Departamento Estadística y Matemàtica Aplicada, Universidad de Almería, Campus de La Cañada, 04120 Almería, Spain. Email address: hmartinezp@terra.es

**** Email address: jmruizgo@um.es
} 
parallel and series systems, and the new better than used of the second order $(\mathrm{NBU}(2))$ and the new worst than used of the second order (NWU(2)) ageing classes were given by Franco et al. (2001).

A similar study has been carried out for some stochastic orders. For results in this direction and references on the subject, the reader is referred to Belzunce et al. (2001) and Boland et al. (1998), (2002).

Recently Li and Yam (2005) proposed the following reversed question. Given an ageing property for the system, do the components have the same ageing property? A similar problem is considered for some stochastic orders. In particular, Li and Yam (2005) proved that if a series system with i.i.d. components is IMRL, DMRL, or new worse than used in convex ordering (NWUC) then the components are IMRL, DMRL, or NWUC, respectively. A similar result is proved for parallel systems and the NWU(2) property. Results for the reversed preservation of the right spread and total time on test orders are also proved.

The purpose of this paper is to provide some additional results to those given by Li and Yam (2005). The organization of this paper is the following. In Section 2 we provide the definitions and previous results of interest, and in Section 3 we provide some new results. In this paper we consider nonnegative random variables, with continuous distributions and left extreme of the support 0 (the results can be extended with slight modifications to more general distributions). Given a random variable $X$ and any event $A$, we denote by $\{X \mid A\}$ the random variable $X$ conditioned to the event $A$. The notation $\stackrel{\text { ST }}{=}$, denotes equality in law.

\section{Definitions and previous results}

In this section we provide the definitions of the ageing classes and stochastic orders that will be considered in the paper. Also, some results that will be used throughout the paper are given. First we give the definitions of the increasing convex and increasing concave orders.

Definition 2.1. Given two nonnegative random variables $X$ and $Y$, with distribution functions $F$ and $G$, respectively, we say that

(i) $X$ is less than $Y$ in the increasing convex order, denoted by $X \leq_{\text {icx }} Y$ or alternatively $F \leq_{\text {icx }} G$, if

$$
\int_{x}^{+\infty}(1-F(u)) \mathrm{d} u \leq \int_{x}^{+\infty}(1-G(u)) \mathrm{d} u \text { for all } x \geq 0,
$$

(ii) $X$ is less than $Y$ in the increasing concave order, denoted by $X \leq_{\text {icv }} Y$ or alternatively $F \leq_{\text {icv }} G$, if

$$
\int_{0}^{x}(1-F(u)) \mathrm{d} u \leq \int_{0}^{x}(1-G(u)) \mathrm{d} u \text { for all } x \geq 0 .
$$

For applications and properties of these orders in reliability, economics, and risk theory, the reader is referred to Müller and Stoyan (2002), and Shaked and Shanhtikumar (2007).

Now we proceed to give the definitions of the ageing classes considered in the paper. For general references about definitions, properties, and applications, the reader is referred to Barlow and Proschan (1981), Gertsbakh (1989), and Lai and Xie (2006).

Definition 2.2. Let $X$ be a nonnegative random variable with absolutely continuous distribution function $F$ and density function $f$. We say that $X$ has a DFR, or alternatively $X$ or $F$ has a $\mathrm{DFR}$, if the failure rate $r(t) \equiv f(t) /(1-F(t))$ is decreasing for all $t \geq 0$. 
For the DFR ageing class, it is easy to prove the following result that will be used later.

Lemma 2.1. Let $X$ be a nonnegative random variable with absolutely continuous distribution function $F$, density function $f$, and hazard rate $r$. If $X$ has a DFR then $f$ is decreasing.

Proof. It follows from the equality $f(t)=r(t)(1-F(t))$.

Definition 2.3. Let $X$ be a nonnegative random variable with continuous distribution $F$. We say that $X$ has an IFRA or a DFRA, or alternatively $X$ or $F$ has an IFRA or a DFRA, if

$$
\frac{R(t)}{t}=\frac{-\ln (1-F(t))}{t} \text { is increasing or decreasing in } t>0,
$$

respectively.

To finish we consider some ageing notions which are defined through the concept of residual life. Given a nonnegative random variable $X$ and given $t \geq 0$, the residual lifetime at time $t$ is the random variable $X_{t} \equiv\{X-t \mid X \geq t\}$, which represents the remaining lifetime of a unit which has survived up to time $t$.

Definition 2.4. Let $X$ be a nonnegative random variable. We say that $X$ is NWUE, or alternatively that $X$ or $F$ is NWUE, if $\mathrm{E}\left(X_{t}\right) \geq \mathrm{E}(X)$ for all $t \geq 0$.

This ageing class can be characterized as follows; see Abouammoh and El-Neweihi (1986).

Lemma 2.2. Let $X$ be a nonnegative random variable with continuous distribution function $F$. Then $X$ is NWUE if and only if

$$
\int_{0}^{t}(1-F(u)) \mathrm{d} u \leq \frac{F(t)}{(1-F(t))} \int_{t}^{\infty}(1-F(u)) \mathrm{d} u \text { for all } t \geq 0 .
$$

Definition 2.5. Let $X$ be a nonnegative random variable with continuous distribution function $F$. We say that $X$ has an IMRL, or alternatively that $X$ or $F$ has an IMRL, if $\mathrm{E}\left(X_{t}\right)$ is increasing in $t \geq 0$.

A characterization of this class is the following; see Abouammoh and El-Neweihi (1986).

Lemma 2.3. Let $X$ be a nonnegative random variable with absolutely continuous distribution function $F$ and density function $f$. Then $X$ has an IMRL if and only if

$$
\frac{f(t)}{(1-F(t))^{2}} \int_{t}^{\infty}(1-F(u)) \mathrm{d} u \geq 1 \text { for all } t \geq 0 .
$$

Cao and Wang (1991) considered the following ageing class by comparisons with residual lives in the increasing convex order previously defined.

Definition 2.6. Let $X$ be a nonnegative random variable with continuous distribution function $F$. We say that $X$ is new better than used in the increasing convex order, or alternatively that $X$ or $F$ is NBUC, if

$$
X \geq \text { icx } X_{t} \text { for all } t \geq 0 .
$$

We conclude this section with a technical result that will be used in the next section; see Barlow and Proschan (1981). 
Lemma 2.4. Let $W(x)$ be a Lebesgue-Stieltjes measure not necessarily positive. If

(a) $h(x)$ is a nonnegative increasing function and $\int_{t}^{\infty} \mathrm{d} W(x) \geq 0$ for all $t \geq 0$, then

$$
\int_{0}^{\infty} h(x) \mathrm{d} W(x) \geq 0
$$

(b) $h(x)$ is a nonnegative decreasing function and $\int_{0}^{t} \mathrm{~d} W(x) \geq 0$ for all $t \geq 0$, then

$$
\int_{0}^{\infty} h(x) \mathrm{d} W(x) \geq 0
$$

Throughout this paper, we consider series and parallel systems. Given a set of $n$ components with random lifetimes $X_{1}, X_{2}, \ldots, X_{n}$, a series system formed by the $n$ components is a system that fails if one of the components fails. The random lifetime of a series system will be denoted by $X_{(1, n)}$, and clearly $X_{(1, n)}=\min \left\{X_{1}, X_{2}, \ldots, X_{n}\right\}$. If the random lifetimes are i.i.d. with common distribution function $F$ then the distribution function of $X_{(1, n)}$ is given by

$$
1-(1-F(t))^{n} \quad \text { for all } t \geq 0 .
$$

A parallel system fails when all the components have failed, and in this case the random lifetime of the parallel system is given by $X_{(n, n)}=\max \left\{X_{1}, X_{2}, \ldots, X_{n}\right\}$. If the random lifetimes are i.i.d. with common distribution function $F$ then the distribution function of $X_{(n, n)}$ is given by

$$
F^{n}(t) \text { for all } t \geq 0
$$

\section{Reversed preservation properties}

In this section we give several results for the reversed preservation of some stochastic orders and ageing classes for series and parallel systems. We first consider results for the increasing convex and increasing concave orders.

\subsection{Reversed preservation of stochastic orders}

First we prove the reversed preservation of the increasing convex order for series systems.

Theorem 3.1. Let $X_{1}, \ldots, X_{n}$ be a set of $n$ i.i.d. random variables with distribution function $F$, and let $Y_{1}, \ldots, Y_{n}$ be another set of $n$ i.i.d. random variables with distribution function $G$. If $X_{(1, n)} \leq_{\mathrm{icx}} Y_{(1, n)}$, then $F \leq_{\mathrm{icx}} G$, that is, $X_{i} \leq_{\mathrm{icx}} Y_{i}$ for $i=1, \ldots, n$.

Proof. We need to prove that

$$
\int_{t}^{\infty}(1-F(x)) \mathrm{d} x \leq \int_{t}^{\infty}(1-G(x)) \mathrm{d} x \quad \text { for all } t \geq 0 .
$$

From the assumptions and (2.1), we have

$$
\int_{t}^{\infty}\left((1-G(x))^{n}-(1-F(x))^{n}\right) \mathrm{d} x \geq 0 \quad \text { for all } t \geq 0 .
$$

This inequality can be written as

$$
\int_{t}^{\infty}(\bar{G}(x)-\bar{F}(x))\left(\bar{G}^{n-1}(x)+\bar{G}^{n-2}(x) \bar{F}(x)+\cdots+\bar{F}^{n-1}(x)\right) \mathrm{d} x \geq 0 \quad \text { for all } t \geq 0,
$$


where $\bar{F}=1-F$ and $\bar{G}=1-G$ denote the survival functions associated to $F$ and $G$, respectively. It is easy to see that the function

$$
h(x)=\left(\bar{G}^{n-1}(x)+\bar{G}^{n-2}(x) \bar{F}(x)+\cdots+\bar{F}^{n-1}(x)\right)^{-1}
$$

is increasing and nonnegative. Therefore, by Lemma 2.4(a) and taking

$$
\mathrm{d} W(x)=(\bar{G}(x)-\bar{F}(x))\left(\bar{G}^{n-1}(x)+\bar{G}^{n-2}(x) \bar{F}(x)+\cdots+\bar{F}^{n-1}(x)\right) \mathrm{d} x,
$$

we obtain

$$
\begin{gathered}
\int_{t}^{\infty} h(x)(\bar{G}(x)-\bar{F}(x))\left(\bar{G}^{n-1}(x)+\bar{G}^{n-2}(x) \bar{F}(x)+\cdots+\bar{F}^{n-1}(x)\right) \mathrm{d} x \\
=\int_{t}^{\infty} \bar{G}(x) \mathrm{d} x-\int_{t}^{\infty} \bar{F}(x) \mathrm{d} x \geq 0 \quad \text { for all } t \geq 0 .
\end{gathered}
$$

Therefore, we obtain (3.1), that is, $F \leq_{\text {icx }} G$.

Next we prove a similar result for parallel systems and the increasing concave order.

Theorem 3.2. Let $X_{1}, \ldots, X_{n}$ be $n$ i.i.d. random variables with distribution function $F$, and let $Y_{1}, \ldots, Y_{n}$ be another set of $n$ i.i.d. random variables with distribution function $G$. If $X_{(n, n)} \leq_{\mathrm{icv}} Y_{(n, n)}$ then $F \leq_{\mathrm{icv}} G$, that is, $X_{i} \leq_{\mathrm{icv}} Y_{i}$ for $i=1, \ldots, n$.

Proof. In this case we need to prove the following inequality:

$$
\int_{0}^{t}(1-F(x)) \mathrm{d} x \leq \int_{0}^{t}(1-G(x)) \mathrm{d} x \text { for all } t \geq 0 .
$$

By hypothesis and from (2.2), we find that

$$
\int_{0}^{t}\left(F^{n}(x)-G^{n}(x)\right) \mathrm{d} x \geq 0 \quad \text { for all } t \geq 0 .
$$

This inequality can be written as

$$
\int_{t}^{\infty}(F(x)-G(x))\left(F(x)^{n-1}+F^{n-2}(x) G(x)+\cdots+G^{n-1}(x)\right) \mathrm{d} x \geq 0 \quad \text { for all } t \geq 0 .
$$

Now it is easy to show that the function

$$
h(x)=\left(F^{n-1}(x)+F^{n-2}(x) G(x)+\cdots+G^{n-1}(x)\right)^{-1}
$$

is decreasing and nonnegative. Therefore, by Lemma 2.4(b) and considering

$$
\mathrm{d} W(x)=(F(x)-G(x))\left(F^{n-1}(x)+F^{n-2}(x) G(x)+\cdots+G^{n-1}(x)\right) \mathrm{d} x,
$$

we obtain

$$
\int_{0}^{t}(F(x)-G(x)) \mathrm{d} x=\int_{0}^{t}((1-G(x))-(1-F(x))) \mathrm{d} x \geq 0 \quad \text { for all } t \geq 0 .
$$

Therefore, we obtain (3.2), that is, $F \leq_{\text {icv }} G$. 


\subsection{Reversed preservation of ageing properties}

Next we consider several results for reversed preservation of ageing classes. First we consider the reversed preservation of the IMRL class for parallel systems.

Theorem 3.3. Let $X_{1}, \ldots, X_{n}$ be $n$ i.i.d. random variables with absolutely continuous distribution function $F$ and density function $f$. If $X_{(n, n)}$ is IMRL then $F$ is IMRL.

Proof. To prove that $F$ is IMRL, we will consider the characterization given in Lemma 2.1, that is, $F$ is IMRL if and only if

$$
1 \leq \frac{f(t)}{(1-F(t))^{2}} \int_{t}^{+\infty}(1-F(u)) \mathrm{d} u \text { for all } t \geq 0 .
$$

By hypothesis $X_{(n, n)}$ is IMRL, and by Lemma 2.1 and (2.2), we have

$$
1 \leq \frac{n F^{n-1}(t) f(t)}{\left(1-F^{n}(t)\right)^{2}} \int_{t}^{\infty}\left(1-F^{n}(u)\right) \mathrm{d} u \quad \text { for all } t \geq 0 .
$$

Therefore, from (3.3), if

$$
\frac{n F^{n-1}(t) f(t)}{\left(1-F^{n}(t)\right)^{2}} \int_{t}^{\infty}\left(1-F^{n}(u)\right) \mathrm{d} u \leq \frac{f(t)}{(1-F(t))^{2}} \int_{t}^{\infty}(1-F(u)) \mathrm{d} u \quad \text { for all } t \geq 0
$$

then $F$ is IMRL. This inequality follows if we prove the following inequality:

$$
\frac{n F^{n-1}(t) f(t)}{\left(1-F^{n}(t)\right)^{2}}\left(1-F^{n}(u)\right) \leq \frac{f(t)}{(1-F(t))^{2}}(1-F(u))
$$

for all $t \geq 0$ and for all $u \geq t$.

From

$$
1-F^{n}(t)=(1-F(t))\left(1+F(t)+\cdots+F^{n-1}(t)\right),
$$

we find that (3.4) is equivalent to

$$
n F^{n-1}(t)\left(1+F(u)+\cdots+F^{n-1}(u)\right) \leq\left(1+F(t)+\cdots+F^{n-1}(t)\right)^{2}
$$

for all $t \geq 0$ and for all $u \geq t$, or equivalently,

$$
n^{1 / 2} F^{(n-1) / 2}(t)\left(1+F(u)+\cdots+F^{n-1}(u)\right)^{1 / 2} \leq 1+F(t)+\cdots+F^{n-1}(t)
$$

for all $t \geq 0$ and for all $u \geq t$. Given that $\left(1+F(u)+\cdots+F^{n-1}(u)\right)^{1 / 2} \leq n^{1 / 2}$, then (3.4) is true if

$$
0 \leq 1+F(t)+\cdots+F^{n-1}(t)-n F^{(n-1) / 2}(t) \text { for all } t \geq 0,
$$

and this follows from

$$
\begin{aligned}
1+F(t)+\cdots+F^{n-1}(t)-n F^{(n-1) / 2}(t) & \\
& =\sum_{j=0}^{[(n-1) / 2]}\left(1-F^{(n-1) /(2-j)}(t)\right)\left(F^{j}(t)-F^{(n-1) / 2}(t)\right) \geq 0,
\end{aligned}
$$

where $[(n-1) / 2]$ denotes the integer part of $(n-1) / 2$ (see Abouammoh and El-Neweihi (1986)). Therefore, $F$ is IMRL. 
Next we consider a similar result for the DFR ageing class.

Theorem 3.4. Let $X_{1}, \ldots, X_{n}$ be $n$ i.i.d. random variables with absolutely continuous distribution function $F$ and differentiable density function $f$. If $X_{(n, n)}$ has a DFR then $F$ has a DFR.

Proof. Let us prove that $F$ has a DFR, that is, that the hazard rate is decreasing, or equivalently, that

$$
f^{2}(t)+(1-F(t)) f^{\prime}(t) \leq 0 \text { for all } t \geq 0 .
$$

By hypothesis the hazard rate of $X_{(n, n)}$ is decreasing in $t \geq 0$; therefore,

$$
\frac{\left(n(n-1) F^{n-2}(t) f^{2}(t)+n F^{n-1}(t) f^{\prime}(t)\right)\left(1-F^{n}(t)\right)+\left(n F^{n-1}(t) f(t)\right)^{2}}{\left(1-F^{n}(t)\right)^{2}} \leq 0
$$

for all $t \geq 0$. This inequality can be written as follows:

$$
\begin{aligned}
\left(n(n-1) F^{n-2}(t)\left(1-F^{n}(t)\right)+\right. & \left.n^{2}\left(F^{n-1}(t)\right)^{2}\right) f^{2}(t) \\
& +n F^{n-1}(t) f^{\prime}(t)\left(1-F^{n}(t)\right) \leq 0 \quad \text { for all } t \geq 0 .
\end{aligned}
$$

Also, by Lemma 2.1, the hazard rate of $X_{(n, n)}$ is decreasing, that is,

$$
n(n-1) F^{n-2}(t) f^{2}(t)+n F^{n-1}(t) f^{\prime}(t) \leq 0 \quad \text { for all } t \geq 0 ;
$$

therefore, $f^{\prime}(t) \leq 0$.

From

$$
\left(1-F^{n}(t)\right)=(1-F(t))\left(1+F(t)+\cdots+F^{n-1}(t)\right) \leq n(1-F(t))
$$

and (3.6), we obtain

$$
\begin{aligned}
& \left(n^{2} F^{n-2}(t)\left(1-F^{n}(t)\right)-n^{2} F^{n-2}(t)(1-F(t))+n^{2} F^{2 n-2}(t)\right) f^{2}(t) \\
& +n^{2} F^{n-1}(t)(1-F(t)) f^{\prime}(t) \leq 0 \quad \text { for all } t \geq 0,
\end{aligned}
$$

which is equivalent to

$$
f^{2}(t)+(1-F(t)) f^{\prime}(t) \leq 0 \quad \text { for all } t \geq 0
$$

therefore, (3.5) is true and $F$ has a DFR.

To complete the results for parallel systems we consider a result for the NWUE ageing class.

Theorem 3.5. Let $X_{1}, \ldots, X_{n}$ be $n$ i.i.d. random variables with continuous distribution function F. If $X_{(n, n)}$ is NWUE then $F$ is NWUE.

Proof. In this case to prove that $F$ is NWUE, we consider the characterization given in Lemma 2.2, that is, $F$ is NWUE if and only if

$$
\int_{0}^{t}(1-F(u)) \mathrm{d} u \leq \frac{F(t)}{1-F(t)} \int_{t}^{\infty}(1-F(u)) \mathrm{d} u \quad \text { for all } t \geq 0 .
$$


By hypothesis and Lemma 2.2, we have

$$
\int_{0}^{t}\left(1-F^{n}(u)\right) \mathrm{d} u \leq \frac{F^{n}(t)}{1-F^{n}(t)} \int_{t}^{\infty}\left(1-F^{n}(u)\right) \mathrm{d} u \text { for all } t \geq 0,
$$

and given that

$$
\int_{0}^{t}(1-F(u)) \mathrm{d} u \leq \int_{0}^{t}\left(1-F^{n}(u)\right) \mathrm{d} u \text { for all } t \geq 0,
$$

if we prove that

$$
\frac{F^{n}(t)}{1-F^{n}(t)} \int_{t}^{\infty}\left(1-F^{n}(u)\right) \mathrm{d} u \leq \frac{F(t)}{1-F(t)} \int_{t}^{\infty}(1-F(u)) \mathrm{d} u \quad \text { for all } t \geq 0
$$

then (3.7) is true.

By proving that

$$
\frac{F^{n}(t)}{1-F^{n}(t)}\left(1-F^{n}(u)\right) \leq \frac{F(t)}{1-F(t)}(1-F(u))
$$

for all $t \geq 0$ and for all $u \geq t$, the result follows.

Considering

$$
1-F^{n}(t)=(1-F(t))\left(1+F(t)+F^{2}(t)+\cdots+F^{n-1}(t)\right),
$$

we find that (3.8) is equivalent to

$$
\frac{F^{n}(t)\left(1+F(u)+\cdots+F^{n-1}(u)\right)}{(1-F(t))\left(1+F(t)+\cdots+F^{n-1}(t)\right)}(1-F(u)) \leq \frac{F(t)}{1-F(t)}(1-F(u))
$$

for all $t \geq 0$ and for all $u \geq t$, that is

$$
F^{n-1}(t)\left(1+F(u)+\cdots+F^{n-1}(u)\right) \leq 1+F(t)+\cdots+F^{n-1}(t)
$$

for all $t \geq 0$ and for all $u \geq t$. This inequality is true, as can be easily seen; therefore, (3.8) is true, and $F$ is NWUE.

To finish we consider two reversed preservation results for series systems. First we consider the IFRA and DFRA ageing classes.

Theorem 3.6. Let $X_{1}, \ldots, X_{n}$ be $n$ i.i.d. random variables with continuous distribution function $F$. The series system $X_{(1, n)}$ is IFRA or DFRA if and only if $F$ is IFRA or DFRA, respectively.

Proof. Clearly, $X_{(1, n)}$ is IFRA or DFRA if and only if

$$
\frac{R_{X_{(1, n)}}(t)}{t}=\frac{-\ln (1-F(t))^{n}}{t}=\frac{-n \ln (1-F(t))}{t} \text { is increasing or decreasing in } t,
$$

respectively, which is equivalent to

$$
\frac{R(t)}{t}=\frac{-\ln (1-F(t))}{t} \text { is increasing or decreasing in } t,
$$

respectively, that is, $F$ is IFRA or DFRA, respectively. 
Next we prove a reversed preservation property for the NBUC ageing class. To obtain this result, the following result will be considered. This result can be found in Pellerey and Petakos (2002).

Lemma 3.1. (Pellerey and Petakos (2002).) Given a nonnegative integer $n$, then

$$
\left(\min \left\{X_{1}, \ldots, X_{n}\right\}\right)_{t} \stackrel{\mathrm{sT}}{=} \min \left\{\left(X_{1}\right)_{t}, \ldots,\left(X_{n}\right)_{t}\right\} .
$$

Theorem 3.7. Let $X_{1}, \ldots, X_{n}$ be $n$ i.i.d. random variables with continuous distribution function F. If $X_{(1, n)}$ is NBUC then $F$ is NBUC.

Proof. Recall that $F$ is NBUC if

$$
\int_{t}^{\infty} \bar{F}_{t}(x) \mathrm{d} x \leq \int_{t}^{\infty} \bar{F}(x) \mathrm{d} x \quad \text { for all } t \geq 0 .
$$

By hypothesis

$$
\left(\min \left\{X_{1}, \ldots, X_{n}\right\}\right)_{t} \leq_{\text {icx }} \min \left\{X_{1}, \ldots, X_{n}\right\} \text { for all } t \geq 0
$$

and, from Lemma 3.1, this is equivalent to

$$
\int_{x}^{\infty} \bar{F}_{t}^{n}(y) \mathrm{d} y \leq \int_{x}^{\infty} \bar{F}^{n}(y) \mathrm{d} y \text { for all } t, x \geq 0 .
$$

This inequality can be written as

$$
\begin{aligned}
\int_{x}^{\infty} & \left(\bar{F}^{n}(y)-\bar{F}_{t}^{n}(y)\right) \mathrm{d} y \\
& =\int_{x}^{\infty}\left(\bar{F}(y)-\bar{F}_{t}(y)\right)\left(\bar{F}^{n-1}(y)+\bar{F}^{n-2}(y) \bar{F}_{t}(y)+\cdots+\bar{F}_{t}^{n-1}(y)\right) \mathrm{d} y \geq 0 .
\end{aligned}
$$

Now it is easy to see that the function

$$
h(y)=\left(\bar{F}^{n-1}(y)+\bar{F}^{n-2}(y) \bar{F}_{t}(y)+\cdots+\bar{F}_{t}^{n-1}(y)\right)^{-1}
$$

is nonnegative and increasing, and from Lemma 2.4(a) and considering

$$
\mathrm{d} W(y)=\left(\bar{F}(y)-\bar{F}_{t}(y)\right)\left(\bar{F}^{n-1}(y)+\bar{F}^{n-2}(y) \bar{F}_{t}(y)+\cdots+\bar{F}_{t}^{n-1}(y)\right) \mathrm{d} y,
$$

we obtain

$$
\int_{x}^{\infty}\left(\bar{F}(y)-\bar{F}_{t}(y)\right) \mathrm{d} y \geq 0 \text { for all } t, x \geq 0,
$$

that is, $F$ is NBUC.

\section{Acknowledgements}

This research was supported by the Ministerio de Educación y Ciencia under grant MTM2006-12834 and by the Fundación Séneca (CARM 00698/PI/04). 


\section{References}

Авоuammon, A. And El-Neweini, E. (1986). Closure of the NBUE and DMRL classes under formation of parallel systems. Statist. Prob. Lett. 4, 223-225.

Barlow, R. E. And Proschan, F. (1981). Statistical Theory of Reliability and Life Testing. Probability Models. To Begin With, Silver Spring, MD.

Belzunce, F., Ruiz, J. M. And Ruiz, M. C. (2002). On preservation of some shifted and proportional orders by systems. Statist. Prob. Lett. 60, 141-154.

Belzunce, F., Franco, M., Ruiz, J. M. And Ruiz, M. C. (2001). On partial orderings between coherent systems with different structures. Prob. Eng. Inf. Sci. 15, 273-293.

Boland, P. J., Shaked, M. and Shanthikumar, J. G. (1998). Stochastic ordering of order statistics. In Order Statistics; Theory \& Methods (Handbook Statist. 16), eds N. Balakrishnan and C. R. Rao, Elsevier, Amsterdam, pp. 89-103.

Boland, P. J., Hu, T., Shaked, M. and Shanthikumar, J. G. (2002). Stochastic ordering of order statistics. II. In Modeling Uncertainty: An Examination of Stochastic Theory, Methods and Applications, eds M. Dror et al., Kluwer, Boston, MA, pp. 607-623.

Cao, J. and Wang, Y. (1991). The NBUC and NWUC classes of life distributions. J. Appl. Prob. 28, 473-479. (Correction: 29 (1992), 753.)

Esary, J. D. And Proschan, F. (1963). Relationship between system failure rate and component failure rates. Technometrics 5, 183-189.

Esary, J. D., Marshall, A. W. And Proschan, F. (1970). Some reliability applications of the hazard transform. SIAM J. Appl. Math. 18, 849-860.

Franco, M., Ruiz, J. M. and Ruiz, M. C. (2001). On closure of the IFR(2) and NBU(2) classes. J. Appl. Prob. 38, 235-241.

Franco, M., Ruiz, J. M. AND RuIz, M. C. (2003). A note on closure of the ILR and DLR classes under formation of coherent systems. Statist. Papers 44, 279-288.

GertsbakH, I. B. (1989). Statistical Reliability Theory. Marcel Dekker, New York.

Hendi, M. I., Mashrour, A. F. And Montasser, M. A. (1993). Closure of the NBUC class under formation of parallel systems. J. Appl. Prob. 30, 975-978.

LaI, C. D. AND XIE, M. (2006). Stochastic Aging and Dependence for Reliability. Springer, New York.

LI, X. AND YAM, R. (2005). Reversed preservation properties of some negative aging conceptions and stochastic orders. Statist. Papers 46, 65-78.

Li, X., Li, Z. And Jing, B. (2000). Some results about the NBUC class of life distributions. Statist. Prob. Lett. 49, 229-237.

Müller, A. And Stoyan, D. (2002). Comparison Methods for Stochastic Models and Risks. John Wiley, Chichester.

Pellerey, F. and Petakos, K. (2002). On closure property of the NBUC class under formation of parallel systems. IEEE Trans. Reliability 51, 452-454.

Ross, S. M. (1972). Introduction to Probability Models. Academic Press, New York.

Shaked, M. and Shanthikumar, J. G. (2007). Stochastic Orders. Springer, New York. 